Merino Mañueco, S. (2018). Formación del profesorado con respecto al error y a su corrección en las producciones escritas de alumnos de L2: conocimientos, aptitudes y actitudes. Revista Electrónica Interuniversitaria de Formación del Profesorado, 21(2), 155-166.

\title{
Formación del profesorado con respecto al error y a su corrección en las producciones escritas de alumnos de L2: conocimientos, aptitudes y actitudes
}

\author{
Susana Merino Mañueco \\ Universidad de Valladolid
}

\section{Resumen}

El error y su corrección se revelan como elementos clave en la enseñanza-aprendizaje de una segunda lengua (L2). Los factores generadores de errores, tras haber sido objeto de numerosos estudios sobre la enseñanza-aprendizaje de L2, comienzan a manifestarse como parte imprescindible de la competencia profesional docente. Es parte de la responsabilidad de las instituciones académicas encargadas de la formación del profesorado centrarse en la corrección de errores en la perspectiva de que los profesores cuenten con unos conocimientos, unas aptitudes y unas actitudes orientadas a la mejora de la corrección y al feedback en la enseñanza-aprendizaje de L2. En el presente trabajo nos proponemos realizar una revisión teórica en torno a los tres componentes de la competencia profesional docente, a saber: los conocimientos, las aptitudes y las actitudes de los profesores de L2 en relación al error y a su corrección en las producciones escritas.

\section{Palabras clave}

Error; corrección; enseñanza-aprendizaje; L2.

\section{Teacher training in error and its correction in the written productions of L2 students: knowledge, skills and attitudes}

\section{Contacto:}

Susana Merino Mañueco, susana.merino.manueco@uva.es. Facultad de Educación y Trabajo Social. Universidad de Valladolid. Paseo de Belén, 1. Campus Miguel Delibes, 47011, Valladolid.

Artículo vinculado a proyecto de investigación financiado por la Consejería de Educación. Junta de Castilla y León. Orden EDU/332/2017. Licencia por estudios curso 2017-2018. 


\begin{abstract}
The concepts of error and error correction have been considered a focus of attention in the teaching and learning of a second language (L2). One of the main goals in L2 teaching and learning research is to identify error-causing factors in student written productions. The identification of these factors is therefore becoming an essential part of professional teaching competence. As a consequence, the responsible academic institutions for teacher training must provide teachers the necessary training on error correction and feedback. In this paper we intend to conduct a theoretical review around the three components of professional teaching competence: knowledge, skills and attitudes of L2 teachers regarding error and error correction in written productions.
\end{abstract}

\title{
Key words
}

Error; correction; teaching and learning; L2.

\section{Introducción}

Dada la importancia de la corrección de errores, la formación del profesorado en torno a esta se perfila como componente necesario tanto desde una perspectiva teórica como práctica. Refiriéndose a los manuales escolares y a las guías didácticas que los acompañan, instrumento considerado tradicional para la enseñanza-aprendizaje de lenguas, Burt (1975) ya venía destacando que estos no ofrecen una formación directa y explícita para una corrección eficaz, con lo cual se impone como necesidad una formación al respecto. En este acercamiento, el autor hacía hincapié en que la formación del profesorado esté enfocada hacia los posibles errores ya que, en su opinión se impone como imprescindible "to be prepared to handle the variety of errors that inevitably occur in student speech and writing" (Burt, 1975, p. 53).

La existencia de numerosos estudios realizados en torno a la enseñanza-aprendizaje orientados a identificar los factores generadores de errores en L2 resaltan, según encontramos en Stockman y Pluut (1999), la obligación de incluir dichos factores dentro de la competencia profesional docente del profesor de L2 o como ellos mismos destacan "a major goal of second-language acquisition research is to identify factors that influence speaker/listener errors" (p. 185).

Así es como, se empieza a hablar de unas exigencias por parte de las instituciones de enseñanza de una formación de los profesores de L2 orientada en torno a la identificación, clasificación, explicación y corrección de errores.

El conocimiento del propio proceso de corrección de errores supondrá, en palabras de Torijano Pérez (2004), saber adecuarse a la realidad de la clase, a la personalidad del alumno, al tipo de error y a la frecuencia con que se repite haciendo al alumno:

Consciente de su capacidad: que no piense que ésta es nula porque recibe una corrección cada dos palabras, pero que tampoco se considere acreedor al nivel propio del nativo porque el profesor no le haya hecho notar ninguna de sus desviaciones. (Torijano Pérez, 2004, pp. 72-73)

Desde el contexto educativo rumano, Constantinescu (2011) insistía en la necesidad, por parte del profesor, de tener un conocimiento preciso de los errores de sus alumnos. En este sentido, propone exponerlos durante los primeros días de clase a una serie de actividades 
en las que se les solicitará la elaboración de varios textos en L2. A partir de la corrección y posterior análisis de dichas producciones escritas, el profesor podrá elaborar un corpus de errores, lo cual le permitirá posteriormente "de répertorier les erreurs et de les classer par rubriques: phonétique, morphosyntaxe, semantique, etc" (Constantinescu, 2011, p. 85). Esta clasificación de los errores más frecuentes le ayudará a seleccionar las actividades "susceptibles de résoudre les difficultés d'apprentissages rencontrés par ses éleves" (Constantinescu, 2011, p. 85).

La misma opinión la encontramos en Ferris (2004) quien, desde el contexto educativo norteamericano, afirmaba que los profesores deben prepararse para poder llevar a cabo un tratamiento de errores adecuado. Una formación que incluya no solo aspectos teóricos relacionados con la gramática de la L2 (cuyo conocimiento profundo les permitirá poder aportar una explicación sobre la norma gramatical correspondiente), sino también aspectos prácticos en torno a la localización de los errores y su posterior interpretación.

Nos situamos en la línea de la mencionada autora por cuanto que el profesor debe contar con la formación necesaria para poder ser capaz de proporcionar al alumno el feedback que acompañará a la corrección de errores. La forma de aproximarse al alumno y de hacerle llegar dicho feedback, sin que este consiga despistarlo o desanimarlo durante la elaboración de su producción escrita implica, en palabras de Ferris (2004) "some careful decisionmaking on the part of the teacher which considers the students' needs and backgrounds and the instructional context" (p. 59). A esta necesidad de formación del profesor en torno a la corrección de errores y al feedback, la citada autora añade como requisito imprescindible de la competencia profesional docente el hecho de que los profesores de L2 sepan transmitir al alumno la importancia de que sean ellos mismos los que desarrollen sus propias estrategias de revisión y de corrección de las producciones escritas para detectar y, posteriormente, aprender de sus propios errores.

\section{Conocimientos, aptitudes y actitudes del profesor ante el error y su corrección}

Los conocimientos junto con las aptitudes y las actitudes del profesor sobre el error son los componentes que entran a formar parte de la formación profesional docente. Desde las políticas lingüísticas europeas manifestadas en determinados documentos de referencia se ha intentado conceder una atención a la formación del profesor orientada al error y a su corrección. Es el caso del Marco Común Europeo de Referencia para las lenguas: aprendizaje, enseñanza, evaluación (MCER) (Ministerio de Educación, Cultura y Deporte [MECD], 2002) el cual plantea la necesidad de que los profesores de $L 2$ cuenten con un conocimiento preciso sobre las faltas y los errores de los alumnos estableciendo una clara diferencia entre estos. Del mencionado documento europeo extraemos la idea de proporcionar un tratamiento distinto para cada uno de los casos proponiendo determinadas claves que sustenten el desarrollo profesional docente:

a) el profesor debería corregir inmediatamente todos los errores y faltas;

b) se debería fomentar sistemáticamente la corrección inmediata por parte de un compañero y, de este modo, conseguir erradicar los errores;

c) se deben señalar y corregir todos los errores cuando esto no interfiera en la comunicación (por ejemplo: separando el desarrollo de la corrección del desarrollo de la fluidez); 
d) los errores no sólo deberían corregirse, sino también analizarse y explicarse en el momento adecuado;

e) las faltas que son sólo simples deslices se deberían pasar por alto, pero los errores sistemáticos deben erradicarse;

f) se deben corregir los errores sólo cuando interfieren en la comunicación;

g) los errores se deberían aceptar como «interlengua de transición» y no deberían tenerse en cuenta. (MECD, 2002, p. 154)

\section{El papel del profesor: conocimientos sobre el error y su corrección}

Al hablar de conocimientos sobre la corrección de errores, debemos hacer alusión al papel que el profesor debe asumir como guía y orientador de los alumnos en su trabajo. Así es como, lejos de asumir el papel principal debe favorecer la autonomía del alumno. Encontramos este mismo concepto en autores como Blanco y Garrido (2013); Erdocia (2016); Fabián Martín-Arroyo (2016); Gómez Pazos y Hernando Gómez (2016); Loaiza Villalba y Galindo Martínez (2014); Sánchez Godoy y Casal Madinabeitia (2016) quienes ponen de manifiesto la necesidad de que los alumnos adquieran un determinado grado de responsabilidad sobre su propio proceso de enseñanza-aprendizaje. Por tanto, se impone que los conocimientos teóricos y prácticos de los profesores de L2 orientados a la corrección de errores deben girar en torno a las técnicas de corrección en las que el alumno pueda participar de una forma activa. Así pues, impulsar y motivar a los alumnos para que sean ellos mismos los que con la ayuda del profesor realicen el ejercicio de detección, revisión y autocorrección se configura como parte esencial de la competencia profesional docente (Barranco Izquierdo \& Guillén Díaz, 2017; Dumbrăvescu, 2017; Sanz Trigueros, 2015) orientada a la corrección de errores.

Recurrimos también a otro documento europeo Las competencias clave del profesorado de lenguas segundas y extranjeras lanzado por el Instituto Cervantes en el año 2012. El mencionado documento propone a los profesores de L2 "crear y desarrollar oportunidades de aprendizaje para todos los alumnos" (Instituto Cervantes, 2012, p. 13) promoviendo que estos tomen conciencia de sus propias necesidades comunicativas. Según el documento, los profesores son los responsables de crear situaciones de aprendizaje en las que sean los mismos alumnos quienes evalúen la tarea realizada identificando y corrigiendo sus propios errores.

Nos mostramos de acuerdo con Cassany (1993) y con Peters (1984) por cuanto que asociamos los conocimientos del profesor en torno al error y a la corrección a todo un proceso de reflexión que debe acompañar al acto profesional didáctico de la corrección de errores. Así pues, Cassany (1993, p. 29), definiéndose a sí mismo como "maestro-corrector" que ha pasado muchos años "señalando errores a ciegas, sin saber exactamente para qué lo hacía” (p. 29), propone la idea de reflexionar y buscar cuál es la información que se quiere transmitir al alumno-receptor de nuestros comentarios o correcciones y, al mismo tiempo, "definir la utilidad didáctica" (p. 29) que se da a la corrección. Peters (1984), a su vez, destacaba la necesidad de entender la corrección como un proceso marcado por la reflexión del profesor y, en su opinión:

Reflection upon correct and incorrect (error analysis) actions or results comprises an important quality of didactical activity. Reflection can occur during all the phases of didactical activity: during planning; after completion of the planning; during the realization; after the realization has taken place. We can divide reflection into three types: pre-reflection; peri-reflection; 
post-reflection. These types of reflection follow one another; that is, they take place before, during and after interactive teaching. (Peters, 1984, p. 22)

En la presente cita observamos que Peters (1984) también hace referencia a la necesidad de llevar a cabo todo este proceso de reflexión no solo al final de la actividad de corrección de errores, sino que, como hemos apuntado anteriormente, dicha reflexión ha de estar presente a lo largo de todo el proceso didáctico. Retenemos por tanto la idea que plantea Peters (1984), respecto a la continuidad de la reflexión y asociamos los tres momentos mencionados en su cita con los Actos Profesionales Docentes (APD) que forman parte de la responsabilidad docente. Así pues, el momento de pre-reflection correspondería a la fase de planificación, el peri-reflection a la fase de intervención y, finalmente el momento de post-reflection se relacionaría con la fase de evaluación.

Por todo ello, los conocimientos del profesor, desde su vertiente reflexiva, influirán de manera decisiva tanto en las propias aptitudes, entendidas estas como futuras actuaciones llevadas a cabo en torno al error y a su corrección, como también en las actitudes o conductas que acompañan a dicho proceso.

\section{Aptitudes en torno al error y su corrección}

Podemos decir que, en ocasiones, el docente se encuentra ante varias incógnitas relacionadas con la corrección de errores no solo con respecto a su posible utilidad, sino también acerca de qué, cómo y cuándo corregir. Se impone que para llevar a cabo todo el proceso de corrección debe demostrar y poner en práctica una serie de aptitudes. La aptitud, en palabras de Mallart (2004), aparece definida como la "capacidad latente de poder realizar alguna acción o de poder aprender alguna actividad" (p. 76). Sin embargo, Galisson y Coste (1982, p. 42), la entendían en términos de predisposición o "substrat constitutionnel" (p. 42) del sujeto que se manifiesta como "capacité directement mesurable" (p. 42). Se revela, por tanto, como "un ensemble de comportements" (p. 54) que podríamos traducir como una competencia dirigida hacia la corrección de errores.

Volvemos a acudir a los documentos de referencia europeos observando que es el MCER (MECD, 2002) el que lanzaba la idea de incluir los resultados extraídos de la observación y del análisis de errores de los alumnos en la programación general del curso. Asimismo, estos resultados podrán proporcionar orientación para la planificación de las actividades, la secuenciación didáctica y los materiales utilizados. Además, podrán ayudar al profesor en el proceso de evaluación de la enseñanza-aprendizaje de la L2 del alumno (Merino Mañueco, 2016).

En otros documentos europeos de referencia de las lenguas como European Profile for Language Teacher Education - A Frame of Reference (Kelly, Grenfell, Allan, Kriza \& McEvoy, 2004) en el que se presentan los aspectos más representativos de un programa de formación de profesorado no se concede atención a la aptitud del profesor orientada a corregir. Observamos, por tanto, que en dicho documento se han enfocado otros aspectos como el de aportar recursos, actividades orientadas a las nuevas tecnologías de la información, interculturalidad, ciudadanía europea...etc. Sin embargo, no menciona el hecho de dotar a los profesores, durante su formación, de unas aptitudes específicas en torno a la corrección de errores.

Desde el contexto norteamericano, Ferris (2002) señalaba la importancia de que los profesores de L2 estén capacitados para la corrección de errores en L2 afirmando que "before we can prepare our students to cope with errors in their writing, we must prepare ourselves" (p. 48). La autora manifiesta en su estudio el hecho de que muchos de los 
futuros profesores del programa de master del que ella formaba parte, no eran capaces de aportar un análisis adecuado de los errores de las producciones escritas de sus alumnos. Considera, por tanto, que el profesor de L2 no solo necesita contar con aptitudes que le permitan saber escoger de entre una gran variedad de recursos, técnicas y materiales orientados al análisis de errores, sino también contar con un sólido conocimiento de la lengua respaldado de unas aptitudes en torno al análisis efectivo de errores.

Ferris (2002) formulaba y describía cuatro principios fundamentales que debe cumplir todo profesor de L2 en términos de corrección de errores. El primero de ellos alude a la exigencia por parte de las instituciones de que los profesores tengan un dominio de aquellos aspectos gramaticales especialmente problemáticos para los hablantes no nativos; el segundo principio destaca la necesidad de que los profesores puedan ser capaces de reconocer e identificar los errores de las producciones escritas de los alumnos. Como tercer principio, la autora resalta la importancia de la enseñanza de la gramática de la L2 por parte del profesor unida a la necesidad de poseer un conocimiento profundo de lo que implica la enseñanza-aprendizaje de una L2.

Desde el contexto español, Cassany (1993) incluía en su obra un decálogo de recomendaciones respecto a qué, cómo y cuándo corregir los errores con el fin de intentar mejorar la expresión escrita de los alumnos de L2. En primer lugar, propone que se corrija solo lo que el alumno pueda aprender; en segundo lugar, que se lleve a cabo la corrección inmediatamente después de la producción para que el alumno pueda recordar el contexto y el motivo por el cual decidió utilizar una expresión en particular; la tercera recomendación gira en torno a la corrección de las versiones o borradores escritos por el alumno antes de llevar a cabo el texto final ya que dichas producciones aportarán una idea más amplia de las intenciones del alumno. Además, el autor propone al profesor que no sea él quien realice todo el trabajo de corrección, sino que intente crear situaciones de aprendizaje que se presten a la corrección en parejas, en grupos o autocorrección. A esta recomendación añade la de dar instrucciones concretas y prácticas, dejar tiempo en la clase para que los alumnos puedan leer y comentar las correcciones, hablar individualmente con cada alumno, aportar instrumentos que permitan a los alumnos autocorregirse: diccionarios, gramáticas... etc. asegurando la calidad de la corrección. La visión de Cassany (1993) es, en definitiva, la de utilizar la corrección como un recurso didáctico en sí.

Asimismo, tal y como planteaban Cassany (1993) y Ferris (2002), se impone la idea de contar con unas aptitudes relacionadas con la corrección además de un conocimiento profundo respecto al qué, cómo y cuándo corregir los errores de las producciones escritas de sus alumnos de L2, en definitiva, con una formación específica al respecto.

Actitudes ante el error y su incidencia en la enseñanza-aprendizaje de expresión escrita en L2

El término de actitud cuenta con numerosas acepciones. Nos detenemos en la propuesta de Hobbs (2011) sobre las creencias de los profesores de L2, definiéndolas como "ideas, attitudes, assumptions, values and knowledge about teaching and learning that teachers accumulate and bring with them to the classroom" (p. 156) al destacar su importancia en el ámbito de la educación de los profesores de lenguas puesto que "examining them helps language teaching education researchers and educators to understand better the complex nature of the classroom" (Hobbs, 2011, p. 156).

Desde el ámbito de la Didáctica, Galisson y Coste (1982) definían el término actitud como una serie de disposiciones interiores de un individuo con respecto a los objetos, situaciones 
y acontecimientos sujetos a una apreciación valorativa. Dichas disposiciones interiores pueden variar considerablemente, según comentaba Alfieri Lane (1997), de manera que existen: "educators who believe in the positive aspects of error correction and those who think that this practice is extremely harmful and detrimental to the process of acquisition" (p. 7).

Desde el contexto educativo rumano, Constantinescu (2011), en relación a la actitud adoptada por el profesor ante el error, introducía la idea de que "loin de sanctionner l'erreur, mieux vaut la placer au centre de la démarche pédagogique” (p. 84).

Corresponde a las responsabilidades del profesor identificar los errores e integrarlos en el proceso de enseñanza-aprendizaje de una L2, idea que encontramos en Alfieri Lane (1997, p. 16), quien consideraba que, en ese sentido, "educators' beliefs and attitudes toward error correction" han de ser especialmente cuidados y analizados ya que son ellos los encargados de "implement error correction strategies" (p.16).

Durán (2011) indicaba la presencia de tres categorías de profesores en función de la actitud adoptada ante la corrección de errores. La primera de ellas, denominada heavy corrector correspondería a una actitud centrada en corregir todo sin saltarse ningún error. La segunda, non-corrector representa una actitud dirigida a la corrección del menor número de errores posible. Por último, la categoría selective corrector revela una actitud de corrección ante los errores que necesitan ser corregidos.

Refiriéndose a los estudios llevados a cabo respecto a la corrección de errores, Serkan Ülgü y Griffiths (2013, p. 229) destacaban que los profesores no cuentan con una opinión fundamentada en los resultados de estudios empíricos, sino que parten generalmente de unas "intuiciones", según ponen de manifiesto a continuación: “teachers' point of view on error correction options does not go beyond intuitionist ideas rather than being empirically evidenced" (p. 229). Además, esta posición tan poco fundamentada es lo que determina a los profesores a optar por una corrección inmediata tanto de una producción escrita como también oral tal y como lo expresan Serkan Ülgü y Griffiths (2013) en su estudio llevado a cabo en el contexto educativo turco: "teachers want to correct their learners" errors. Teachers are in favor of immediate correction for written or oral errors" (p. 237).

Esto se une a la idea de Truscott (1996) quien, refiriéndose a la corrección gramatical, destacaba la falta de relación directa entre corrección y aprendizaje. Es lo que le determinó a afirmar rotundamente que la corrección gramatical no es efectiva y, por lo tanto "grammar correction is not helpful and can be harmful" (Truscott, 1996, p. 355) desatando una fuerte polémica en el ámbito de la enseñanza-aprendizaje. Asimismo, Truscott (1996, p. 342 ) intentaba dar una explicación a esa popularidad de la corrección gramatical por parte de los profesores. Según él, dichos profesores no consiguen tener una visión de lo que es la interlengua $\mathrm{y}$, sabiendo que esta implica un proceso cognitivo de gran complejidad, prefieren adoptar una visión simple de lo que es el aprendizaje de L2 enfocando su atención en la gramática.

Por esta razón, de acuerdo con Corder (1981), la responsabilidad de todo profesor ha de estar orientada hacia la necesidad de adoptar una actitud más realista ante la producción del alumno estimando necesario "a shift of emphasis in teaching away from a preoccupation with the grammar of the target language towards a concern with communication in the target language" (p. 78).

Es evidente que se pueden encontrar diferentes muestras de actitudes contradictorias hacia la presencia del error. Así pues, el MCER (MECD, 2002) propone una tipología de 
actitudes existentes en torno a las faltas y a los errores de los alumnos, según se puede observar a continuación:

a) los errores y las faltas demuestran el fracaso del aprendizaje;

b) los errores y las faltas demuestran una enseñanza ineficaz;

c) los errores y las faltas demuestran la voluntad que tiene el alumno de comunicarse a pesar del riesgo de equivocarse;

d) los errores son el producto inevitable y pasajero de la interlengua que desarrolla el alumno;

e) las faltas son inevitables en cualquier uso de la lengua, incluyendo el de los hablantes nativos. (MECD, 2002, pp. 153-154)

Nos posicionamos en la perspectiva de Gardner (2010) por cuanto que existe la necesidad por parte del profesor de crear "a relaxed atmosphere in the L2 classroom" (p. 188) que se asociará a una actitud positiva hacia el error y hacia la corrección. Diversos estudios coinciden en señalar el hecho de que un ambiente distendido y de confianza influirá en los alumnos a la hora de expresar sus ideas y opiniones de una manera libre. Según el mencionado autor, esto también implica que los profesores adopten una actitud de tolerancia ante el error y de cercanía hacia el alumno. Una relación cercana entre alumnoprofesor y un ambiente relajado en el aula serán determinantes para motivar al alumno y lograr una predisposición favorable hacia la realización de sus producciones escritas de una manera autónoma.

\section{Discusión y conclusiones}

La valoración del error es entendida como la posición que toma el profesor ante este y el aprovechamiento y/o posible integración dentro del proceso de enseñanza-aprendizaje de L2. Partiendo de dicha valoración, nos centramos en la actitud como un componente que, junto con los conocimientos y las aptitudes, forman parte del concepto de competencia del profesor en torno al error.

Es obligación del profesor, transmitir al alumno la idea de la importancia que tiene la enseñanza y el aprendizaje de la lengua mostrándole los enormes beneficios que podrá encontrar en un futuro si es capaz de adquirir un buen dominio tanto de su lengua materna como de otras lenguas. Es de suma importancia, desde un punto de vista didáctico, que el alumno trabaje su producción escrita de una manera organizada, utilizando un vocabulario rico y matizado ya que, "quien escribe de forma ordenada, con corrección ortográfica y con pulcritud, proyectará siempre una imagen positiva de sí mismo ante los demás" (Ministerio de Educación, Cultura y Deporte [MECD], 2011, Introducción, 2).

El documento lanzado en 2011 por la Secretaría de Estado de Educación y Formación Profesional del Ministerio de Educación, Cultura y Deporte, pone de manifiesto que, los alumnos, especialmente durante la etapa de Educación Primaria "deberán ir mejorando poco a poco en la redacción de textos breves, coherentes y bien organizados" (MECD, 2011, Introducción, 3). Para ello, el profesor deberá solicitar al alumno que produzca una amplia variedad de textos escritos siempre relacionados con temas cercanos a sus intereses particulares con el objetivo de que se lleve a cabo un aprendizaje significativo. 
En una sociedad marcada por el auge evidente de las Nuevas Tecnologías de la Información en la que "la publicidad y la informática han modificado sustancialmente los cánones gráficos y la tecnología de la escritura" (Viñao Frago, 2002, p. 358) se impone conceder una especial atención a la expresión escrita de los alumnos de L2. El uso de redes sociales, programas, y aplicaciones informáticas que hemos incluido en nuestra vida diaria, nos obliga a comunicarnos constantemente a través de textos escritos los cuales, no necesitan ser textos completos y pulidos, sino que cumplen una función meramente comunicativa. Es obvio que, en los mencionados mensajes de texto utilizados en chats y redes sociales se toleran determinados errores (como, por ejemplo, los de tipo ortográfico o de puntuación), pero, por otro lado, existen otros que pueden llevar a confusiones y a una falta de comunicación.

Podemos decir que, a pesar de la omnipresencia de la Nuevas Tecnologías instaurada en nuestra sociedad actual debemos tener en cuenta que todavía se demanda un tipo de escritura comprensible y legible tanto para el emisor como para el receptor o receptores. Consecuentemente, se impone que el profesor siempre procure introducir ejercicios que motiven al alumno para que este mantenga una buena disposición hacia el aprendizaje de la L2, utilizando una metodología que favorezca una actitud positiva (Méndez Santos, 2016) hacia la lengua y hacia la producción escrita. En el aula de lengua en general y en el de L2, en nuestro caso particular, se impone como necesidad desarrollar la expresión escrita como destreza. Sin embargo, toda producción escrita, especialmente en una L2, conlleva implícitamente la idea de cometer errores ya que, a la hora de redactar, el alumno recurre a la interlengua o "sistema transitorio" (Núñez-Méndez, 2001, p. 133) por el que atraviesa el aprendiz en su acercamiento a una competencia en $\mathrm{L} 2$.

Desde esta perspectiva se pone de relieve la necesidad de una formación del profesorado de L2 en torno al error y a su corrección. Así pues, coincidimos con de la Torre et al. (1994) quienes insistían en esta idea ya que dicha formación permitirá al profesor conocer el nivel de competencia en $\mathrm{L} 2$ de los alumnos, adquirir conocimientos sobre el funcionamiento de la L2, y "obtener informaciones sobre las dificultades de aprendizaje más comunes" (p. 92). La formación del profesor, de acuerdo con los mencionados autores ha de estar orientada a la observación y al uso de los instrumentos de diagnóstico de errores. Esta actividad de clasificación y diagnóstico de errores debe realizarse, de acuerdo con de la Torre et al. (1994) de manera conjunta con los propios alumnos, ya que, según los autores, deben "reconocer y descubrir sus propios errores favoreciendo la autogestión de los mismos" (p. 122). Se impone por tanto la necesidad de involucrar a estos en su propio proceso de enseñanza-aprendizaje.

\section{Referencias}

Alfieri Lane, L. G. (1997). Error correction: An investigation of teachers ideas and attitudes. Tesis de Magister no publicada, University of Texas, Austin, Texas, Estados Unidos.

Blanco, M.P. \& Garrido, M.C. (2013). Lenguas de especialidad e interacción oral en el sector turístico: Reflexiones sobre su implicación en el Espacio Europeo de Educación Superior. Revista Electrónica Interuniversitaria de Formación del Profesorado, 16(1), 25---40. DOI: http://dx.doi.org/10.6018/reifop.16.1.179421

Barranco Izquierdo, N. \& Guillén Díaz, C. (2017). Efectos de la mediación oral para la resolución de conflictos comunicativos en aulas de inglés de Educación Primaria con presencia de alumnado extranjero. Revista Electrónica Interuniversitaria de 
Formación del Profesorado, 20(1), 159-172. DOI: http://dx.doi.org/10.6018/reifop.20.1.229271

Burt, M. (1975). Error Analysis in the Adult EFL Classroom. TESOL Quarterly, 9(1), 53-63.

Cassany, D. (1993). Reparar la escritura. Didáctica de la corrección de lo escrito. Barcelona : Graó.

Constantinescu, I. (2011). Dictionnaire pratique de didactique du français langue étrangère. Bucureşti: Fundația România de Mâine.

Corder, S. P. (1981). Error Analysis and Interlanguage. Oxford: Oxford University Press.

de la Torre, S., Mallart, J., Tort, LI., Rajadell, N., Laffitte, R. \& Oliver, C. (1994). Errores y currículo. Tratamiento didáctico de los errores en la enseñanza. Barcelona: Promociones y publicaciones universitarias.

Dumbrăvescu, D. G. (2017). Los manuales escolares y la formación inicial del profesorado de ELE. Valencia: Educalia.

Durán, R. (2011). Guidelines for Error Correction in the EFL Classroom. En S. House, (Coord.), Inglés. Investigación, innovación y buenas prácticas, (pp. 61-67). Barcelona: Graó.

Erdocia, I. (2016). Dimensión contextual y autonomía del aprendiente de lenguas. [Con]textos, 5(20), 35-43. DOI: 10.21774/ctx.v5i20.756

España, Ministerio de Educación, Cultura y Deporte. (2002). Marco Común Europeo de Referencia para las lenguas: aprendizaje, enseñanza, evaluación (MCER). Madrid: Secretaría General Técnica del Ministerio de Educación, Cultura y Deporte y Grupo Anaya.

España, Ministerio de Educación, Cultura y Deporte. (2011). Resolución de la Secretaría de Estado de Educación y Formación Profesional por la que se establece el currículo de la Sección Española del Programa de Estudios Internacionales del Distrito Escolar público del condado de Miami-Dade del estado de Florida (Estados Unidos). Recuperado el 15 septiembre 2017 de

http://www.mecd.gob.es/dms-static/6dab6548-6bb5-4d4d-b2e8-

27b6bffagbof/consejerias-exteriores

Fabián Martín-Arroyo, M.D. (2016). El temido bolígrafo rojo. La elaboración de sistemas de corrección para la expresión escrita. En Lamolda González, M. a Á. (Comp.), La formación y competencias del profesorado de ELE. Actas del XXVI Congreso Internacional ASELE. (pp. 307-316). Granada: Universidad de Granada.

Ferris, D. (2002). Treatment of error in second language student writing. Ann Arbor, MI: University of Michigan Press.

Ferris, D. R. (2004). The "grammar correction" debate in L2 writing: Where are we, and where do we go from here? (and what do we do in the meantime . . .?). Journal of Second Language Writing 13, 49-62. DOI: 10.1016/j.jslw.2004.04.005.

Galisson, R. \& Coste D. (Dirs.) (1982). Dictionnaire de didactique des langues. París: Hachette.

Gardner, R. C. (2010). Motivation and second language acquisition: The socio-educational model. New York, NY : Peter Lang.

Gómez Pazos, M. \& Hernando Gómez, A. (2016). Experiencia docente acerca del uso didáctico del aprendizaje cooperativo y el trabajo de campo en el estudio del fenómeno de influencia social. Revista Electrónica Interuniversitaria de Formación del Profesorado, 19(2), 331---346. DOI: http://dx.doi.org/10.6018/reifop.19.2.206921 
Hobbs, V. (2011). The importance of teachers' thoughts and beliefs. En S. House, (Ed.), Theory and Practice in English Language Teaching. (pp. 147-159). Barcelona: Grao.

Instituto Cervantes. (2012). Las competencias clave del profesorado de lenguas segundas y extranjeras. Recuperado el 22 septiembre 2017 de http://cfp.cervantes.es/imagenes/File/competencias_profesorado.pdf

Kelly, M., Grenfell, M., Allan, R., Kriza, C. \& McEvoy, W. (2004). European Profile for Language Teacher Education - A Frame of Reference. Southampton, UK: University of Southampton.

Loaiza Villalba, N. \& Galindo Martínez, A. (2014). Estilos de aprendizaje de segundas lenguas y formación bilingüe consecutiva en educación primaria, secundaria y superior: hacia un estado del arte. Lenguaje, 42(2), 291-314.

Mallart, J. (2004). Aptitud. En F. Salvador Mata, J. L. Rodríguez Diéguez \& A. Bolívar Botía, (Dirs.), Diccionario enciclopédico de didáctica (p. 76). Málaga: Aljibe.

Méndez Santos, M.C. (2016). La dimensión afectiva en la formación del profesorado de ELE. En Lamolda González, M. ${ }^{a}$ Á. (Comp.), La formación y competencias del profesorado de ELE. Actas del XXVI Congreso Internacional ASELE. (pp. 681-691). Granada: Universidad de Granada.

Merino Mañueco, S. (2016). El análisis de errores en los enfoques actuales de enseñanzaaprendizaje de segundas lenguas. En Lamolda González, M. ${ }^{a}$ Á. (Comp.), La formación y competencias del profesorado de ELE. Actas del XXVI Congreso Internacional ASELE. (pp. 693-698). Granada: Universidad de Granada.

Núñez-Méndez, E. (2001, enero). Corregir o no los errores en la clase de español como LE. Trabajo presentado en el VII Simposium of Social Communication. Proceedings of the VII Simposium of Social Communication. Center of Applied Linguistics: Vol. 2 (pp. 132138). Universidad de Santiago de Cuba, Santiago de Cuba, Cuba.

Peters, J. J. (1984). Teaching: Intentionality, reflection and routines. En Halkes, R. \& Olson, J. K. (Eds.), Teacher thinking. A new perspective on persisting problems in education (pp. 19-34). Lisse, Holanda: Swets and Zeitlinger.

Sánchez Godoy, I. \& Casal Madinabeitia, S. (2016). El desarrollo de la autonomía mediante las técnicas de aprendizaje cooperativo en el aula de L2. Porta Linguarum, 25, 179190.

Sanz Trigueros, J.F. (2015). Del Marco Europeo de las cualificaciones al "European Profiling Grid" como un referencial de empleabilidad de los docentes. En Actas Congreso Internacional Observal 2015. Cualificaciones profesionales y acreditación de competencias. Valladolid: Universidad de Valladolid (en prensa).

Serkan Ülgü, I. S. \& Griffiths, C. (2013). Error correction in language teaching: The teachers' perspective. International Journal of Arts \& Sciences, 6(3), 229-241.

Stockman, I. J. \& Pluut, P. (1999). Segment composition as a factor in the syllabification errors of second-language speakers. Language Learning, 49(1), 185-209.

Torijano Pérez, J.A. (2004). Errores de aprendizaje, aprendizaje de los errores. Madrid: Arco Libros.

Truscott, J. (1996). The case against grammar correction in L2 writing classes. Language Learning,46 (2), 327-369. 
Viñao Frago, A. (2002). La Enseñanza de la Lectura y la Escritura: Análisis Socio-Histórico. Anales de Documentación, 5, 345-359. 